\title{
Paolo Zaccone
}

Università degli Studi di Torino

paolozaccone.studiolegale@gmail.com

\section{Il sistema elettorale italiano dalla nascita della repubblica alla 1. 52/2015}

DOI: http://dx.doi.org/10.12775/SIT.2017.041

\section{Premessa}

Nella presente trattazione si prende in analisi la legislazione elettorale italiana dal secondo dopoguerra ad oggi, ponendo particolarmente l'accento sulle più recenti evoluzioni in materia.

La legislazione elettorale si compone di tre tipi di norme: innanzitutto quelle che definiscono i diritti politici; in secondo luogo vi sono le regole sul sistema elettorale, che stabiliscono i meccanismi attraverso cui i voti espressi dagli elettori si trasformano in seggi parlamentari; infine c'è la legislazione elettorale di contorno, formata dalle regole relative alle modalità di svolgimento delle campagne elettorali, ai modi di finanziamento della politica, al regime delle ineleggibilità e delle incompatibilità parlamentari ${ }^{1}$.

In questa sede, in particolare, viene illustrata l'evoluzione della normativa italiana sul sistema elettorale. Si tratta di una disciplina di rilievo costituzionale, seppure disciplinata da leggi ordinarie: infatti essa è in grado di condizionare il rendimento della forma di governo e di incidere quindi sull'equilibrio tra i poteri ${ }^{2}$.

\footnotetext{
1 R. Bin, G. Pitruzzella, Diritto costituzionale, Giappichelli, Torino 2013, p. 152.

2 F. Pastore, Partiti e movimenti politici nel momento elettorale sotto la vigenza dell'Italicum, www.dirittifondamentali.it (access: 11.05.2015).
} 
Nei sistemi di democrazia rappresentativa, le leggi sul sistema elettorale dovrebbero conciliare due esigenze fondamentali: da una parte assicurare la governabilità, ossia la stabilità politica e la capacità decisionale dell'esecutivo grazie al sostegno di un'idonea maggioranza parlamentare, dall'altra garantire la rappresentatività, ossia una corrispondenza proporzionale tra il numero di voti ottenuti da ogni lista e il numero di seggi effettivamente conseguiti.

Ai fini di una più completa trattazione, nel primo capitolo appare opportuno illustrare in sintesi il diritto costituzionale di voto, nonché l'assetto costituzionale del Parlamento italiano.

Nel secondo capitolo viene illustrato il sistema elettorale adottato in Italia a partire dal 1948. Si tratta in particolare di un sistema elettorale proporzionale, rimasto in vigore per oltre quarant'anni e undici tornate elettorali. Poiché questa legge garantiva un alto grado di proporzionalità, il forte frazionamento politico veniva riprodotto fedelmente in Parlamento. Per la formazione di un esecutivo era perciò fondamentale un compromesso tra una pluralità di forze politiche.

Il terzo capitolo è dedicato al sistema elettorale prevalentemente maggioritario adottato nel 1993 per garantire una maggiore stabilità di governo e superare l'eccessivo frazionamento politico. Si promuoveva dunque un modello bipartitico, nel quale i due partiti non avrebbero più dovuto rappresentare differenti ideologie, ma avrebbero ora dovuto contrapporsi in relazione alle proposte in campo economico, fiscale, amministrativo-organizzativo dell'apparato statale. La pluralità politica non venne tuttavia eliminata.

Nel quarto capitolo viene illustrata la legge elettorale adottata nel 2005. Questa introduceva un sistema proporzionale con premio di maggioranza alla coalizione o alla singola lista più votata, al fine di assicurare una maggior stabilità all'esecutivo.

Nel quinto capitolo vengono analizzati i profili di illegittimità costituzionale della legge elettorale n. 270/2005, pronunciati dalla sentenza n. 1/2014 della Corte Costituzionale.

Nel sesto capitolo vengono delineati i tratti caratteristici della legge elettorale n. 52/2015. Questa prevede un sistema proporzionale a doppio turno a correzione maggioritaria, con premio di maggioranza per la lista che avesse ottenuto il $40 \%$ dei voti validi 
al primo turno, o che in alternativa avesse vinto il turno di ballottaggio.

Nel settimo e ottavo capitolo vengono esposti le possibili conseguenze derivanti dall'applicazione della legge 52/2015, nonché i suoi eventuali motivi di illegittimità costituzionale.

Infine, nei capitoli nono e decimo vengono illustrati la legge di revisione costituzionale del 2016 e i suoi profili problematici. Questa legge costituzionale propone una radicale modifica dell'assetto istituzionale, con il superamento del bicameralismo perfetto.

\section{Il diritto di voto e il parlamento nella Costituzione italiana}

L'Italia nel dopoguerra assumeva una forma di governo Repubblicana e una nuova Costituzione. La Costituzione repubblicana contiene alcuni principi generali relativi alla materia elettorale. Si tratta in particolare delle norme relative all'area dei diritti politici, all'elettorato attivo, a quello passivo.

Tali disposizioni racchiudono innanzitutto alcuni principi comuni a tutti i sistemi di democrazia rappresentativa contemporanei ${ }^{3}$. In particolare, l'art. 48 comma 1 Cost. afferma il principio del suffragio universale, in base al quale "la titolarità del diritto di voto è riconosciuta a tutti i cittadini, uomini e donne, che hanno raggiunto la maggiore età”. Il comma 2 dichiara che il voto è personale e eguale, ogni elettore infatti pesa allo stesso modo. Inoltre la Costituzione garantisce la libera espressione della propria preferenza nonché la libera, imparziale e oggettiva formazione della propria opinione. A garanzia della scelta libera da interferenze, il voto deve essere altresì segreto. Infine, il diritto di voto può essere limitato esclusivamente per incapacità civile, per effetto di sentenza penale irrevocabile oppure nei casi di indegnità morale indicati dalla legge come prevede il comma 4 dell'art. 48 Cost. Si tratta di principi oramai introdotti in tutti i sistemi democratici.

${ }^{3}$ R. Bin, G. Pitruzzella, op. cit., pp. 152 ss. 
Al contrario, tra questi ordinamenti sussistono differenze rilevanti in relazione all'organizzazione di governo, cioè all'assetto istituzionale. Tali differenze derivano dalle diverse esperienze storiche di ogni paese e dalle successive scelte di amministrazione del territorio.

Sicuramente negli ordinamenti democratici, il momento principale di identificazione tra Stato apparato e corpo sociale è rappresentato dagli organi parlamentari; tuttavia, sono differenti le scelte di legislazione elettorale che stabiliscono i meccanismi attraverso cui i voti espressi dagli elettori vengono convertiti in seggi parlamentari.

L'assetto istituzionale differisce tra i vari paesi in relazione alle scelte ordinamentali effettuate dal Costituente: pertanto, in alcuni paesi l'organo assembleare è monocamerale, avendo la funzione di rappresentare la concezione unitaria e indivisibile della sovranità nazionale, oltre che, dal punto di vista più pratico, di garantire una maggior efficacia e celerità dell'organo legislativo.

Altri paesi hanno preferito la strutturazione dell'organo parlamentare in due assemblee distinte. Questo modello fa riferimento ad una tradizione storica in base alla quale una camera aveva la funzione di rappresentare gli interessi della classe aristocratica, l'altra invece rappresentava la volontà della classe borghese.

Il superamento della suddivisione della società in stati, avvenuto in seguito alla Rivoluzione Francese, determinava l'affermazione di un corpo sociale unitario. Tuttavia molti stati mantenevano in vita la seconda camera, delegandole la rappresentanza di interessi diversi nel mutato assetto politico della comunità statuale.

L'esempio più significativo è fornito dalle Camere Alte dei paesi federali, le quali rappresentano gli interessi degli Stati membri. Nei paesi regionali esse rappresentano gli interessi delle comunità locali. In altri paesi ancora, la Camera Alta è portatrice di particolari interessi, come quelli delle categorie socio-professionali (caso dell'Irlanda e della Slovenia) ${ }^{4}$. Nella Costituzione repubblicana del 1948, l'Italia optava per il mantenimento del sistema bicamerale.

${ }^{4}$ D. E. Tosi, "Forme di governo", in: Diritto costituzionale dei paesi dell'Unione Europea, a cura di E. Palici Di Suni, Cedam, Padova 2007, pp. 26 ss. 
Tuttavia si tratta di un bicameralismo perfetto: le due camere svolgono le medesime funzioni e i suoi membri rappresentano gli stessi interessi, quelli nazionali. Gli unici elementi distintivi tra le due ali del Parlamento sono strutturali, e risiedono nelle modalità di formazione delle due camere.

Innanzitutto queste hanno una consistenza numerica differente. La Camera dei deputati è composta da 630 deputati. Nel Senato siedono invece 315 senatori. Inoltre il Presidente della Repubblica può nominare cinque senatori a vita (art. 59 Cost.). In secondo luogo sono previste età diverse per essere eletti deputati e senatori, rispettivamente 25 e 40 anni. Sono altresì stabilite età differenti per poter eleggere deputati e senatori: hanno diritto di eleggere i deputati tutti i cittadini italiani che abbiano raggiunto la maggior età; i senatori sono invece eletti a suffragio universale diretto dagli elettori che abbiano superato il venticinquesimo anno di età (art. 58 comma 1). Il testo originario della Costituzione, all'art. 60, stabiliva che la Camera dei Deputati fosse eletta per cinque anni, il Senato della Repubblica invece per sei anni. Tuttavia la legge di revisione costituzionale 2/1963 rimuoveva questa differenza e stabiliva una durata analoga, di anni cinque, per entrambi i rami del Parlamento. Relativamente ai meccanismi di elezione della Camera dei Deputati e del Senato venivano adottate due distinte leggi che introducevano due formule elettorali differenti tra loro. In tal modo avrebbe dovuto determinarsi una diversa composizione tra i due rami del Parlamento. Tuttavia le soluzioni adottate non portarono ad una diversificazione strutturale tra le due camere, ma determinarono una sostanziale corrispondenza tra esse.

Nel corso degli anni i diversi interventi legislativi hanno modificato il sistema di elezione delle Camere ma, fino ad oggi, la composizione del Senato è rimasta essenzialmente speculare a quella della Camera Bassa.

Nel corso della legislatura attualmente in carica sono state realizzate due importanti riforme istituzionali: l'adozione di una nuova legge elettorale a seguito della dichiarazione di illegittimità costituzionale della precedente, nonché la legge di revisione costituzionale volta a differenziare la struttura e le funzioni del Senato. 


\section{Il sistema elettorale italiano nel dopoguerra: la repubblica dei partiti}

Dopo la fine dell'esperienza bellica nel ' 45 e la caduta del fascismo, l'operazione di ricostruzione dello Stato italiano veniva realizzata da quei movimenti che avevano condotto la lotta contro il nazifascismo. Queste forze politiche modificarono l'intero assetto costituzionale del paese, anche rispetto a quello dello Stato liberale pre-fascista. L'emblema del rinnovamento era rappresentato dal mutamento della forma di Stato da monarchico a repubblicano. Veniva inoltre adottata una nuova Costituzione che risultava un compromesso tra le istanze, gli interessi, e i valori delle diversa componenti politiche presenti nel paese. Infatti la società italiana era attraversata da profonde fratture e da un sistema politico fortemente polarizzato dal punto di vista ideologico. L'esigenza primaria di tutte le forze era quindi quella di garantire la reciproca sopravvivenza di correnti politiche ed ideologiche inizialmente molto distanti e di favorire la ricerca dell'accordo in luogo della contrapposizione che avrebbe potuto sfociare in esiti dannosi per il sistema.

Pertanto si optò per il modello di democrazia consociativa, il quale incentivava l'accordo tra i principali partiti al fine di condividere il controllo del potere politico. I partiti a livello elettorale competevano quindi ciascuno per conto proprio, per conquistare i seggi parlamentari che attestavano le forze politiche di cui ciascuno disponeva. Dopo le elezioni i partiti usavano la rispettiva forza politica per negoziare tra di loro e raggiungere dei compromessi politici. Tale modello era volto quindi a garantire un equilibrato rapporto di forza in Parlamento e veniva raggiunto con l'adozione di un sistema elettorale proporzionale ${ }^{5}$.

In particolare, la legge per l'elezione della Camera dei Deputati (D.p.r. 361/1957) configurava una formula elettorale proporzionale: essa garantiva una sostanziale corrispondenza tra la percentuale

${ }^{5}$ R. Bin, G. Pitruzzella, op. cit., pp. 130 ss., e C. Fusaro, Le regole della transizione - La nuova legislazione elettorale italiana, Il Mulino 1995, pp. 19 ss. 
di voti ricevuti da ciascun partito e la quota di seggi conseguiti. Per garantire un elevato indice di proporzionalità, il territorio nazionale veniva suddiviso in circoscrizioni di dimensioni molto ampie. $\mathrm{Si}$ trattava più precisamente di 32 circoscrizioni, all'interno di ognuna delle quali venivano eletti quasi venti deputati. Essendo dunque molto elevato il numero di seggi da ripartire in ogni circoscrizione, anche i partiti più piccoli avevano la possibilità di ottenere qualche seggio. Tale sistema elettorale garantiva a tutte le forze politiche la possibilità di sopravvivere, evitava la concentrazione di troppo potere nelle forze maggioritarie, incentivava la ricerca dell'accordo e della mediazione.

L'elezione del Senato avveniva invece secondo un sistema elettorale differente, formalmente maggioritario, ma che tuttavia determinava risultati sostanzialmente corrispondenti a quelli della Camera dei Deputati. Lart. 57 della Costituzione, fin dalle sue origini, stabiliva che il Senato della Repubblica fosse eletto su base regionale. Ad ogni Regione veniva attribuito un certo numero di seggi, tenendo conto della popolazione ivi residente.

Le forze politiche avevano convenuto di adottare un sistema maggioritario con suddivisione del territorio di ogni Regione in un numero di collegi corrispondente a quello dei senatori da eleggere in quella Regione: venivano quindi istituiti tanti piccoli collegi uninominali, in ognuno dei quali avrebbe dovuto essere eletto il candidato che avrebbe conseguito più voti. Tuttavia, nel testo definitivo della legge 6 febbraio 1948 n. 29, veniva inserito un emendamento che prevedeva la necessità di raggiungere il quorum del $65 \%$ delle preferenze per poter essere eletti. I seggi non assegnati per il mancato raggiungimento del quorum, venivano ripartiti con metodo proporzionale su base regionale. Una volta stabilito il numero di seggi spettante ad ogni partito, questi venivano assegnati ai singoli candidati in base alla percentuale conseguita da ciascuno. A causa dell'elevato quorum richiesto e del grande equilibrio tra le forze politiche, l'attribuzione del seggio direttamente nel collegio fu un evento del tutto eccezionale ${ }^{6}$. I senatori vennero dunque solitamente eletti con il metodo proporzionale su esposto. In questo

\footnotetext{
${ }^{6}$ In oltre quarant'anni e undici tornate elettorali, soltanto quaranta sena-
} 
modo era possibile l'elezione di più candidati nello stesso collegio e di nessuno in altri collegi. Inoltre, nonostante l'aumento del numero dei senatori, culminato con la riforma costituzionale del 1963, quando i senatori raggiunsero il numero attuale di 315, il legislatore non aumentò il numero dei collegi. Questi infatti continuavano ad essere 238, come stabiliva la legge 6 febbraio 1948 n. 29: i 77 seggi in più venivano senz'altro destinati ad essere attribuiti con il riparto proporzionale su base regionale. Pertanto, si può affermare che l'elezione dei senatori con metodo maggioritario all'interno di collegi uninominali restò soltanto sulla carta. Solitamente i senatori venivano eletti con metodo proporzionale, in maniera analoga a quella prevista per la Camera dei deputati ${ }^{7}$.

\section{Superamento del parlamentarismo compromissorio: il sistema maggioritario del '93}

La fase del parlamentarismo compromissorio venne superata con il referendum elettorale del 1993. In quest'occasione il popolo si espresse con una larghissima maggioranza a favore dell'abrogazione di alcune norme della legge elettorale del Senato, tra cui l'elevato quorum. Il sistema elettorale per il Senato si trasformava in senso prevalentemente maggioritario-uninominale anche nella sostanza oltre che nella forma. Con il referendum il corpo elettorale esprimeva soprattutto un chiarissimo indirizzo politico a favore di una trasformazione maggioritaria del sistema elettorale. Tuttavia, a causa di dissidi interni ai partiti tradizionali della democrazia italiana, il Parlamento incontrò gravi difficoltà nell'approvare una riforma elettorale. Il risultato finale fu rappresentato da due leggi differenti (il cosiddetto "Mattarellum") ${ }^{8}$ : la legge di riforma del sistema elet-

tori vennero eletti direttamente nel collegio, avendo raggiunto il quorum del $65 \%$ delle preferenze.

7 Ibidem, pp. 32 ss., e R. Bin, G. Pitruzzella, op. cit., pp. 162-167 ss.

8 Il nome Mattarellum è stato attribuito a queste due leggi elettorali dal politologo Prof. Giovanni Sartori, rifacendosi alla tradizione del diritto di usare i nomi latini. Questa denominazione è dovuta al nome del relatore delle leggi, Sergio Mattarella. 
torale del Senato (L. 276/1993) e la legge di riforma del sistema di elezione della Camera dei deputati (L. 277/1993). Queste hanno previsto un sistema misto, prevalentemente maggioritario, in cui il $75 \%$ del totale dei seggi veniva attribuito in collegi uninominali con maggioritario a turno unico, mentre il restante $25 \%$ era ripartito con il metodo proporzionale. La disciplina per l'elezione della Camera dei deputati prevedeva che 475 seggi (poco più del $75 \%$ ) fossero attribuiti in altrettanti seggi uninominali, nei quali veniva eletto il candidato che otteneva il maggior numero di voti. I restanti 143 seggi (il 25\% circa, meno i 12 seggi assegnati alla circoscrizione estero con legge costituzionale 1/2001) venivano attribuiti con metodo proporzionale tra più liste concorrenti, all'interno di 26 circoscrizioni elettorali, a ciascuna delle quali era attribuito un certo numero di seggi in proporzione alla popolazione residente. Innanzitutto veniva stabilita una soglia di sbarramento del $4 \%$, per cui non ottenevano seggi parlamentari le liste che non avessero conseguito tale percentuale di voti a livello nazionale. Inoltre, si applicava lo scorporo, meccanismo consistente nel sottrarre dal conteggio dei voti totali di una lista nella parte proporzionale i voti ottenuti dai candidati collegati alla medesima lista che erano eletti nei collegi uninominali con il sistema maggioritario.

Relativamente all'elezione del Senato della Repubblica, 232 seggi (poco più del $75 \%$ ) venivano assegnati in collegi uninominali con metodo maggioritario, mentre i restanti 77 seggi (25\% circa, visto che con la legge costituzionale 1/2001 sei seggi venivano destinati alla circoscrizione estero) venivano attribuiti con metodo proporzionale nell'ambito di ciascuna Regione. Il Senato continuava infatti ad essere eletto su base regionale e ad ogni Regione era attribuito un numero di seggi anche in proporzione alla popolazione residente ${ }^{9}$. Il territorio di ciascuna Regione era ripartito in collegi uninominali pari al 75\% dei seggi assegnati alla Regione. Per l'assegnazione dei restanti seggi, ciascuna Regione era costituita in un'unica cir-

9 Lart. 57 comma 3 della Costituzione stabilisce che ogni regione debba avere almeno sette senatori. Le uniche eccezioni sono rappresentate dalla Valle d'Aosta, che ha un solo seggio, e dal Molise, che ne ha due: in queste due regioni non c'era spazio per i seggi attribuiti in ragione proporzionale. 
coscrizione elettorale ${ }^{10}$. Inoltre queste leggi elettorali tentavano di semplificare il frammentato sistema partitico italiano. In particolare intendevano spingere le forze politiche verso una logica bipartitica.

Tuttavia non si realizzava un effettivo bipartitismo, in quanto i partiti di ciascuna coalizione concludevano accordi preventivi per la scelta di chi candidare in ciascun collegio uninominale, ripartendo le candidature tra le componenti della coalizione in rapporto alla forza politica ritenuta propria di ciascun partito. In questo modo la frammentazione dei partiti sopravviveva e ciascuna forza della coalizione manteneva, grazie agli accordi politici, un certo numero di parlamentari. L'elettore, esprimendo una scelta per la coalizione nel suo collegio, non poteva far altro che votare per l'unico candidato presentato da quella coalizione, indipendentemente da quale fosse il partito di appartenenza. Questo espediente garantiva la permanenza della pluralità politica, tanto che, nonostante le leggi elettorali volte a promuovere un sistema bipartitico, nelle legislature elette con le leggi del '93 si registrava un elevatissimo numero di gruppi parlamentari. E' infatti illusorio, oltre che sbagliato, pensare che il numero di partiti possa essere ridotto attraverso una disciplina elettorale. Il pluralismo partitico e, di conseguenza, quello parlamentare sono il riflesso del pluralismo del corpo sociale, culturale, ideologico e politico dell'Italia ${ }^{11}$.

\section{L. 270/2005: il ritorno al proporzionale}

Con la legge n. 270 del 21 dicembre 2005 (il cosiddetto "Porcellum") ${ }^{12}$ il sistema elettorale maggioritario veniva abbandonato a discapito di un sistema nuovamente proporzionale. Si trattava tuttavia di un proporzionale impuro, comprensivo altresì di elementi finalizzati

${ }^{10}$ Le uniche due eccezioni erano appunto rappresentate dalla Valle d'Aosta e dal Molise, costituite esclusivamente in collegi uninominali.

11 C. Fusaro, op. cit., pp. 59 ss.

12 Rifacendosi alla tradizione del diritto di usare i nomi latini, il politologo Prof. Giovanni Sartori attribuì il soprannome Porcellum a questa legge poiché il suo relatore, Roberto Calderoli, in un'intervista televisiva definì questa stessa legge "una pocata". 
a rendere più selettivo l'accesso al Parlamento e numericamente più forti le rappresentanze parlamentari che sostenevano l'esecutivo. Questo sistema elettorale era perciò notevolmente differente rispetto al proporzionale puro vigente fino al 1993: l'esperienza del parlamentarismo compromissorio era oramai superata e questa legge, come la precedente, era funzionale al rafforzamento del bipolarismo.

Tuttavia era altresì tesa a rafforzare le singole identità partitiche secondo quanto tipicamente avviene nei sistemi proporzionali, superando la logica bipartitica che la legge elettorale del '93 avrebbe voluto introdurre. Guesto nuovo sistema incentivava perciò ogni partito politico a creare una propria lista autonoma e a collegarsi ad altre liste per formare una coalizione, con preventiva indicazione di un capo coalizione e di un programma comune. Veniva quindi mantenuto l'assetto bipolare introdotto con la riforma del '93, nonché la sostanziale investitura popolare del Presidente del consiglio, tipica delle cosiddette "democrazie immediate"13. Venivano tuttavia tutelate e rafforzate anche le singole forze politiche.

Gli elementi che riducevano la proporzionalità, salvaguardando il carattere tendenzialmente bipolare della competizione elettorale, erano la clausola di sbarramento e il premio di maggioranza. La prima determinava una partecipazione alla ripartizione dei seggi soltanto delle coalizioni di liste o delle singole liste non riunite in coalizione che avessero superato una certa soglia di voti validi. Invece il premio di maggioranza garantiva che la coalizione o la lista singola più votata avessero la maggioranza assoluta in Parlamento.

Anche questa legge adottava regole differenti tra l'elezione della Camera dei deputati e l'elezione del Senato. Tale differenza si fondava ancora sull'art. 57 comma 1 Cost., in base al quale in Senato l'attribuzione dei seggi avviene su base regionale. Perciò in Senato, alla coalizione di liste (o alla lista) che avesse ottenuto il maggior

${ }^{13}$ La struttura formale del potere politico è basata sul rapporto di fiducia tra Governo e Parlamento e perciò l'elettore non vota formalmente per il capo del Governo, bensì per i candidati ai seggi parlamentari. Tuttavia, poiché il leader che vince le elezioni è nominato Primo ministro, quando l'elettore vota per un candidato nel collegio per l'elezione del Parlamento, in realtà sceglie una maggioranza ed il leader di quella maggioranza. 
numero di voti in una certa Regione venivano attribuiti dei seggi aggiuntivi affinché tale coalizione acquisisse il 55\% dei seggi assegnati alla Regione. I restanti seggi venivano ripartiti tra le altre forze politiche che avessero raggiunto la soglia di sbarramento prevista $^{14}$, proporzionalmente al numero di voti validi ottenuti.

Per la Camera dei deputati i seggi erano attribuiti ad ogni coalizione di liste (o ad ogni lista) che avesse raggiunto la soglia di sbarramento prevista ${ }^{15}$, proporzionalmente al numero voti validi ottenuti. Tuttavia, attraverso il premio di maggioranza, la coalizione di liste (o la lista) che avesse ottenuto il maggior numero di voti validi a livello nazionale, avrebbe ottenuto una quota aggiuntiva di seggi, fino al raggiungimento di 340 seggi (circa 55\% dei seggi totali). Il territorio italiano veniva suddiviso in ventisette circoscrizioni plurinominali assegnatarie di un numero di seggi variabili a seconda della popolazione ivi residente; a queste si aggiungeva la circoscrizione uninominale della Valle d'Aosta.

Inoltre questa legge, in applicazione alla riforma costituzionale del 2001, introduceva la novità della circoscrizione estero, nella quale venivano eletti dodici deputati e sei senatori. Un altro elemento di rilievo era rappresentato dalla lista bloccata, per cui l'elettore votava per una delle liste in competizione ma non poteva esprimere alcuna preferenza per i candidati, cosicché questi ri-

14 Per poter partecipare all'attribuzione di seggi in Senato, doveva essere conseguita una clausola di sbarramento a livello regionale, che corrispondeva a: - per le coalizioni di liste, almeno il $20 \%$ dei voti validi espressi se almeno una lista della coalizione avesse conseguito il 3\% dei voti validi espressi; - per le singole liste, almeno l'8\% dei voti validi espressi; - per le liste appartenenti a coalizioni che non avessero raggiunto la percentuale richiesta, almeno l' $\%$ dei voti validi espressi. Sussistevano inoltre particolari soglie di sbarramento a tutela delle minoranze linguistiche riconosciute.

${ }^{15}$ Per poter partecipare all'attribuzione di seggi nella Camera dei deputati, doveva essere conseguita una clausola di sbarramento a livello nazionale, che corrispondeva a: - per le coalizioni di liste, almeno il 10\% dei voti validi espressi se almeno una lista della coalizione avesse conseguito il $2 \%$ dei voti validi espressi; - per le singole liste, almeno il 4\% dei voti validi espressi; - per le liste appartenenti a coalizioni che non avessero raggiunto la percentuale richiesta, almeno il 4\% dei voti validi espressi. Sussistevano inoltre particolari soglie di sbarramento a tutela delle minoranze linguistiche riconosciute. 
sultavano eletti sulla base dell'ordine stabilito al momento della presentazione della lista ${ }^{16}$. L'impossibilità degli elettori di esprimere le preferenze portava i partiti in una posizione di maggior controllo: l'elezione dei parlamentari dipendeva infatti completamente dalle scelte e dalle graduatorie stabilite dai partiti. Inoltre la preventiva indicazione del capo della coalizione focalizzava l'attenzione e il voto sul leader.

Con il superamento del sistema proporzionale puro e del voto ideologico ai grandi partiti tradizionali, emerge infatti la figura di un leader, di una personalità in grado di aggregare intorno a sé un elevato numero di consensi: la campagna elettorale stessa viene totalmente incentrata su questo soggetto ${ }^{17}$.

\section{La dichiarazione \\ di illegittimita' costituzionale della 1. 270/2005: sent. 1/2014 della cort. cost.}

In seguito all'adozione della legge elettorale n. 270/2005, un gruppo di avvocati propose un ricorso dinnanzi a numerosi Tribunali italiani di primo grado, convenendo in giudizio la Presidenza del Consiglio dei ministri e il Ministero dell'interno. In particolare si trattava di azioni di accertamento della pienezza del diritto di voto, diritto politico di rilevanza primaria, come previsto dalla legge elettorale. Il giudizio arrivò davanti alla Corte di Cassazione, che rimise la questione di legittimità costituzionale alla Corte Costituzionale. Parte ricorrente, in particolare, deduceva che nelle elezioni per la Camera dei deputati e per il Senato della Repubblica svoltesi successivamente all'entrata in vigore della legge n. 270 del 2005, ogni cittadino aveva esercitato il diritto di voto secondo le modalità configurate dalla predetta legge, contrarie ai principi costituzionali

${ }^{16}$ Questa legge elettorale prevedeva anche le candidature multiple: i candidati potevano presentarsi contemporaneamente in più circoscrizioni e, ove eletti in più di una di esse, scegliere per l'una piuttosto che per l'altra.

17 R. Bin, G. Pitruzzella, op. cit., pp. 166-171. 
del voto "personale ed eguale, libero e segreto" (art. 48, secondo comma, Cost.) ed "a suffragio universale e diretto" (artt. 56, primo comma e 58, primo comma, Cost.). Pertanto, chiedeva alla Corte di dichiarare che il suo diritto di voto non poteva essere esercitato in modo libero e diretto, secondo le modalità previste e garantite dalla Costituzione e dal protocollo 1 della CEDU, e quindi chiedeva di ripristinarlo secondo modalità conformi alla legalità costituzionale.

La Corte costituzionale accoglieva le istanze dei ricorrenti. Innanzitutto dichiarava l'illegittimità del premio di maggioranza previsto alla Camera dei deputati in quanto questo non era subordinato al raggiungimento di una soglia minima di voti e, quindi, trasformava una maggioranza relativa di voti, potenzialmente anche molto modesta, in una maggioranza assoluta di seggi ${ }^{18}$. Tale meccanismo era manifestamente irragionevole, in quanto distorsivo dei risultati del voto e foriero di un'eccessiva sovra-rappresentanza della lista che avesse conseguito la maggioranza relativa dei suffragi.

Si ledeva inoltre il principio fondamentale di eguaglianza del voto (art. 48, secondo comma, Cost.): in particolare in un sistema proporzionale, seppure impuro, non si sarebbe dovuto arrivare ad una diseguale valutazione del "peso" del voto "in uscita", ai fini dell'attribuzione dei seggi. Le norme sul premio di maggioranza, pur perseguendo un obiettivo di rilievo costituzionale, qual è quello della stabilità del governo del Paese e dell'efficienza dei processi decisionali nell'ambito parlamentare, per come sono state formulate nella presente legge recavano grave pregiudizio ad altri interessi e valori costituzionalmente protetti, quali l'eguaglianza del voto e la proporzionalità della rappresentanza democratica. Analogamente, dovevano ritenersi illegittime le norme relative al Senato. Queste attribuivano un premio di maggioranza assoluta in ambito regionale alla lista (o coalizione di liste) che avesse ottenuto un numero maggiore di voti rispetto alle altre liste, in difetto del raggiungimento di una soglia minima. Nella specie si evidenzia che, oltre a determinare un difetto di proporzionalità e di rappresentanza democratica, l'attribuzione del premio di maggioranza su scala regionale era al-

18 Il partito che avesse ottenuto più voti a livello nazionale, avrebbe conseguito 340 seggi nella Camera dei deputati. 
tresì inidoneo al raggiungimento dell'obiettivo perseguito, quello di un'adeguata stabilità della maggioranza parlamentare e del governo. La maggioranza che si costituiva in Senato era infatti il risultato casuale di una somma di premi regionali, che avrebbero potuto rovesciare il risultato ottenuto dalle liste o coalizioni di liste su base nazionale e favorire la formazione di maggioranze parlamentari non coincidenti nei due rami del Parlamento, pur in presenza di una distribuzione del voto nell'insieme sostanzialmente omogenea. Ciò rischiava di compromettere sia il funzionamento della forma di governo parlamentare delineata dalla Costituzione repubblicana, nella quale il Governo deve avere la fiducia delle due Camere (art. 94, primo comma, Cost.), sia l'esercizio della funzione legislativa, che l'art. 70 Cost. attribuisce collettivamente alla Camera ed al Senato.

Guesta disciplina legislativa, in definitiva, violava manifestamente i principi di proporzionalità, ragionevolezza e di eguaglianza del voto. Infine, la Corte affermava l'illegittimità costituzionale della soppressione del voto di preferenza. In base a questa legge, l'elettore non poteva esprimere alcuna preferenza, ma disponeva soltanto di un voto per la scelta di una lista di partito. Era il partito che designava i candidati e ne stabiliva l'ordine di presentazione nella lista. Questi erano numerosi e difficilmente conoscibili dagli elettori in ragione dell'ampiezza delle circoscrizioni elettorali. Inoltre, non veniva soddisfatta l'aspettativa dell'elettore per cui i candidati venissero eletti nel rispetto di un ordine di lista, vista la possibilità di candidarsi in numerose circoscrizioni (candidature multiple) e la facoltà dell'eletto di optare per altre circoscrizioni sulla base delle indicazioni del partito. Pertanto, nel momento in cui esprimeva la propria preferenza per una lista, il cittadino sceglieva in blocco anche tutti i numerosi candidati in essa elencati, che non avesse avuto modo di conoscere e valutare ${ }^{19}$. Una simile disciplina pri-

19 La previsione di liste bloccate in questa maniera è irrazionale e illegittima, a differenza dei sistemi caratterizzati da liste bloccate solo per una parte dei seggi, o di altri caratterizzati da circoscrizioni elettorali di dimensioni territorialmente ridotte, nelle quali il numero dei candidati da eleggere sia talmente esiguo da garantire l'effettiva conoscibilità degli stessi e con essa l'effettività della scelta e la libertà del voto (al pari di quanto accade nel caso dei collegi uninominali). 
vava l'elettore di ogni margine di scelta dei propri rappresentanti, scelta che era al contrario totalmente rimessa ai partiti: il voto era sostanzialmente "indiretto".

Questa disciplina normativa, tuttavia, non era in linea con i principi costituzionali. I partiti, infatti, non avrebbero potuto sostituirsi al corpo elettorale, in quanto l'art. 67 Cost. presuppone che il mandato parlamentare sia conferito direttamente dagli elettori. Inoltre la presente norma violerebbe gli artt. 56, primo comma, e 58, primo comma, Cost., relativi all'elezione a suffragio universale e diretto rispettivamente dei deputati e dei senatori, nonché l'art. 117, primo comma, Cost., in relazione all'art. 3 del protocollo 1 della CEDU, che riconosce al popolo il diritto alla "scelta del corpo legislativo". Infine, sottraendo all'elettore la facoltà di scegliere l'eletto, si violava il principio stabilito dall'art. 48, secondo comma Cost., secondo il quale il voto avrebbe dovuto essere libero e personale. In definitiva, la totalità dei parlamentari veniva eletta senza la scelta effettiva da parte dei cittadini, alterando il rapporto di rappresentanza tra elettori ed eletti previsto costituzionalmente. Infine la Corte Costituzionale affermava l'ammissibilità delle questioni di legittimità sollevate, pur rientrando le leggi elettorali nel novero delle leggi costituzionalmente necessarie: non sarebbe infatti possibile tollerare la vigenza di norme incostituzionali relative al funzionamento di un sistema democratico-rappresentativo.

Pertanto, la presente pronuncia di illegittimità costituzionale non mirava a eliminare dall'ordinamento l'intera legge n. 270/2005, né a sostituirla con un'altra eterogenea, operando scelte discrezionali spettanti esclusivamente al legislatore. Questa sentenza, al contrario, ripristinava nella legge elettorale contenuti costituzionalmente obbligati, senza compromettere l'idoneità del sistema elettorale a garantire il rinnovo degli organi costituzionali. La normativa elettorale in vigore in seguito all'intervento della Corte prevedeva perciò un sistema proporzionale senza attribuzione del premio di maggioranza all'interno delle stesse circoscrizioni elettorali, sia per la Camera che per il Senato. Inoltre l'elettore aveva diritto di esprimere un voto di preferenza.

Le elezioni che si sono invece svolte in applicazione delle norme elettorali dichiarate costituzionalmente illegittime costituivano un 
fatto oramai concluso. Per questo motivo, la dichiarazione di incostituzionalità di queste norme non produceva alcun effetto sulle elezioni già avvenute, in applicazione del fondamentale principio della continuità dello Stato, in particolare di tutti gli organi costituzionali, a cominciare dal Parlamento.

Infatti, il principio di retroattività delle sentenze di accoglimento di questa Corte (ai sensi dell'art. 136 Cost.) vale soltanto per i rapporti tuttora pendenti, con conseguente esclusione di quelli esauriti, i quali rimangono regolati dalla legge dichiarata invalida.

\section{La legge elettorale n. 52/2015}

Lo scorso maggio veniva approvata la Legge 6 maggio 2015 n. 52 "Disposizioni in materia di elezione della Camera dei deputati" (il cosiddetto "Italicum") ${ }^{20}$. Questa prevede un sistema proporzionale a doppio turno a correzione maggioritaria, con premio di maggioranza, soglia di sbarramento e cento collegi plurinominali con capilista bloccati. Si tratta di una legge che regola esclusivamente l'elezione della Camera dei deputati a partire dal primo luglio 2016, giorno della sua entrata in vigore. Questa legge è parte di un progetto di riforma più ampio, che include la revisione costituzionale per cambiare profondamente il Senato. Le sue origini risalgono al 26 gennaio 2009, quando veniva presentata alla Camera dei deputati una proposta di legge elettorale ad iniziativa popolare, in quanto si riteneva che la precedente legge stravolgesse la volontà degli elettori e non garantisse sempre governabilità. Tale proposta non venne inizialmente esaminata.

La necessità di una nuova legge elettorale si fece tuttavia sentire fortemente dopo la pronuncia della Corte Costituzionale che dichiarava l'illegittimità costituzionale della Legge n. 270/2005. Infatti, le forze politiche volevano evitare che alle elezioni venture si

20 Seguendo la recente abitudine di soprannominare le leggi elettorali con dei latinismi, il principale promotore di questa legge, Matteo Renzi, la soprannominò Italicum per affermarne l'originalità e la mancata influenza rispetto ai modelli provenienti da altri paesi. 
votasse con il sistema proporzionale puro scaturito dalla sentenza della Consulta, inidoneo a garantire la stabilità di governo. Urgeva perciò la realizzazione di una riforma elettorale, che garantisse la stabilità di governo alla forza politica uscita vincitrice dalle elezioni, nel rispetto tuttavia dei principi costituzionali indicati dalla Consulta nella sentenza 1/2014.

Il 10 dicembre 2013 la Commissione Affari Costituzionali della Camera iniziò ad esaminare la proposta di legge popolare su menzionata e ne fece un testo unificato con altre proposte di legge di iniziativa parlamentare. Inizialmente il disegno di legge conteneva anche le norme relative al Senato. In un secondo momento tuttavia la parte relativa alla Camera Alta venne eliminata, nell'ottica della riforma costituzionale che avrebbe dovuto rendere il Senato non più direttamente elettivo. Dopo tre voti di fiducia, il 4 maggio il testo di legge venne approvato definitivamente dal Parlamento e due giorni dopo venne promulgato dal Presidente della Repubblica. L'8 maggio la nuova legge elettorale veniva pubblicata in Gazzetta Ufficiale.

La versione definitiva dell'Italicum riguarda esclusivamente l'elezione della Camera dei deputati. Si tratta di un sistema proporzionale a doppio turno, che prevede premio di maggioranza e soglie di sbarramento, al fine di garantire e promuovere innanzitutto il principio di governabilità. Il legislatore ha sostanzialmente riproposto il sistema elettorale precedente, impegnandosi a correggere i profili di incostituzionalità presenti nella legge n. 270/2005.

In primo luogo, la nuova legge stabilisce che le liste dei candidati siano presentate in 20 circoscrizioni elettorali suddivise nell'insieme in 100 collegi plurinominali, fatti salvi i collegi uninominali nelle circoscrizioni Valle d'Aosta e Trentino-Alto Adige ${ }^{21}$. I collegi elettorali $^{22}$ hanno dimensioni ridotte, a ognuno è assegnato un numero di seggi compreso tra tre e nove. Le liste sono formate da un ca-

${ }^{21}$ Il Trentino Alto Adige è diviso in otto collegi uninominali, la Valle d'Aosta ne ha uno solo. A differenza che negli altri collegi, al loro interno i seggi non sono assegnati con metodo proporzionale.

22 Ai sensi dell'art. 4 della legge 6 maggio 2015 n. 52, i cento collegi elettorali plurinominali sono stati determinati con decreto legislativo n. 122 del 7 agosto 2015 . 
polista e da un elenco di candidati. Al fine di promuovere le pari opportunità tra donne e uomini nell'accesso alle cariche elettive, i candidati devono essere presentati in ogni lista in ordine alternato per sesso e i capilista dello stesso sesso non possono essere più del 60\% del totale in ogni circoscrizione.

Nessuno può essere candidato in più collegi, neppure in altra circoscrizione, salvo i capilista, che possono essere candidati al massimo in dieci collegi. L'elettore può esprimere fino a due preferenze, per candidati di sesso diverso tra quelli che non sono capolista. Egli non può invece esprimere il proprio voto per il capolista, essendo i capilista bloccati. In secondo luogo, la norma stabilisce che accedano alla ripartizione dei seggi le liste che ottengano, su base nazionale, almeno il $3 \%$ dei voti validi. In ogni caso, sono attribuiti 340 seggi alla lista che ottiene, su base nazionale, almeno il $40 \%$ dei voti validi. Nel caso in cui nessuna lista dovesse raggiungere tale soglia, si effettua un turno di ballottaggio tra le due con il maggior numero di voti al fine dell'attribuzione dei 340 seggi. Nel ballottaggio è esclusa ogni forma di collegamento tra liste o di apparentamento tra i due turni di votazione.

Lattribuzione dei seggi alle liste avviene su base nazionale, proiettando le percentuali ottenute dai partiti a livello nazionale sulle circoscrizioni e collegi, e operando la ripartizione in misura proporzionale al numero di voti ottenuti dalle liste nelle diverse circoscrizioni e collegi. Sono proclamati eletti, fino a concorrenza dei seggi che spettano a ciascuna lista in ogni circoscrizione, dapprima i capolista nei collegi, quindi i candidati che hanno ottenuto il maggior numero di preferenze. I dodici seggi attribuiti nella circoscrizione estero sono eletti con il sistema introdotto dalla legge n. 270 del 2005. Come affermato in precedenza, questa legge elettorale disciplina esclusivamente l'elezione della Camera dei deputati. Nell'ottica del legislatore, essa avrebbe dovuto coordinarsi con la riforma costituzionale del Senato in senso non elettivo. Pertanto la sua entrata in vigore veniva fissata alla data del $1^{\circ}$ luglio 2016 , oltre un anno dopo la sua approvazione, al fine di concedere al legislatore il tempo necessario per realizzare la legge di revisione costituzionale del Senato e impedire che alle successive elezioni si votasse con due leggi elettorali differenti tra le due Camere. 
Tuttavia, ad oggi la legge costituzionale non è in vigore: approvata dal parlamento, sarà oggetto di un referendum costituzionale negli ultimi mesi del $2016^{23}$.

\section{Obiettivi e conseguenze della legge n. 52/2015}

A partire dagli anni '90, gli interventi del legislatore in materia elettorale erano volti a garantire la governabilità, attraverso la previsione di diverse modalità di sovrarappresentazione delle forze politiche maggiori e di sottorappresentazione di quelle minori. Tuttavia, il sistema elettorale deve coniugare nella giusta maniera il principio di governabilità con i principi costituzionali della rappresentatività e della libertà di voto.

Poiché la legge n. 270/2005 non riusciva a soddisfare il bilanciamento di questi principi, la Corte Costituzionale pronunciava l'illegittimità di alcune sue previsioni normative, a seguito della cui eliminazione restava in vigore un sistema proporzionale puro. Questo garantiva un'adeguata rappresentatività delle camere parlamentari, senza assicurare tuttavia il raggiungimento di una maggioranza e di un governo stabile. Pertanto il legislatore proponeva una nuova legge elettorale che potesse finalmente garantire quel principio di governabilità tanto perseguito da oltre vent'anni.

La legge 52/2015 infatti, soddisfa innanzitutto il principio della stabilità di governo: alla parte politica uscita vincitrice dalle elezioni viene attribuita una maggioranza parlamentare ampia, in modo che questa sia in grado di sostenere autonomamente un esecutivo. In particolare la lista che supera la soglia del $40 \%$ dei voti o che in alternativa vince al turno di ballottaggio, ottiene 340 seggi alla Camera dei deputati, la maggioranza assoluta. In questo modo un unico partito ha la maggioranza assoluta dei seggi e può sostenere autonomamente il governo.

23 R. Dickmann, A proposito dell'Italicum. Prime osservazioni sul nuovo sistema elettorale di cui alla legge n. 52 del 6 maggio 2015, www.forumcostituzionale.it (access: 03.06.2015), e F. Pastore, op. cit. 
Attraverso questa previsione normativa, il legislatore intende semplificare il sistema politico italiano, riducendo il numero di protagonisti politici e favorendo il partitismo a discapito della politica delle coalizioni: per ottenere la maggioranza assoluta dei seggi parlamentari è infatti necessario abbandonare la logica di coalizione. Da anni in Italia si tenta di modificare il sistema partitico attraverso la legislazione elettorale: si immagina cioè che una disciplina legislativa delle elezioni possa determinare l'assetto del sistema partitico. Tuttavia è illusorio, oltre che sbagliato, pensare che il sistema politico possa essere modificato attraverso una disciplina elettorale. In particolare l'attribuzione del premio di maggioranza a una lista anziché a una coalizione induce le forze politiche a realizzare liste di coalizione che poi, però, subito dopo le elezioni, potrebbero dare luogo alla ricostruzione di una pluralità di soggetti.

Infatti il pluralismo partitico e, di conseguenza, quello parlamentare sono il riflesso del pluralismo del corpo sociale, culturale, ideologico e politico dell'Italia.

Sarebbe quindi piuttosto opportuno cercare di introdurre norme, sia di rango costituzionale che di rango legislativo, che razionalizzino e stabilizzino la forma di governo parlamentare, cercando di valorizzare in qualche modo questo pluralismo, ossia di coniugare la rappresentatività con la governabilità.

In secondo luogo si evidenzia che la legge 52/2015, pur promuovendo la riduzione del pluralismo partitico, incentiva altresi la corsa individuale dei piccoli partiti. Infatti, la previsione di una soglia di sbarramento del 3\% garantisce una rappresentanza anche alle forze politiche più deboli. Perciò, con questo sistema elettorale potrebbe potenzialmente emergere un parlamento costituito da un partito forte e dominante con il 55\% dei seggi, e da minoranze parlamentari numerose e di scarsa consistenza. Tale condizione non è auspicabile in quanto renderebbe difficile la costituzione di un'opposizione sufficientemente forte da potersi proporre come autorevole punto di riferimento per un'alternativa di governo, tipica invece delle democrazie moderne più avanzate.

Guindi la bassa soglia di sbarramento determina un sostanziale indebolimento della lista che perde al ballottaggio e comunque delle maggiori liste di opposizione. Se infatti la lista vincitrice ottiene il 
$55 \%$ dei seggi parlamentari, il restante $45 \%$ dei seggi deve essere ripartito tra i numerosi protagonisti politici che hanno superato il $3 \%$ dei consensi. Sarebbe stato sicuramente più opportuno innalzare la soglia di sbarramento, in modo da escludere le liste minori e rafforzare il ruolo dell'opposizione ${ }^{24}$.

\section{Profili di incostituzionalita' della} legge elettorale n. 52/2015

La legge elettorale 52/2015 è incentrata sul principio di governabilità: essa ripropone per la Camera un modello simile a quello previsto dalla legge precedente, introducendo però alcuni correttivi indicati dalla sentenza della Corte Costituzionale, con l'obiettivo di bilanciare il principio di stabilità di governo con gli altri principi costituzionali. Tuttavia, poiché tale legge non può prescindere dalla riforma di semplificazione istituzionale, che presuppone in particolare il superamento del bicameralismo perfetto con forte mutamento strutturale e funzionale del Senato, il legislatore avrebbe dovuto occuparsi inizialmente della riforma costituzionale, per poi provvedere all'approvazione di una legge ordinaria attuativa della riforma. Infatti l'efficacia della disciplina proposta dalla legge elettorale è strettamente connessa al superamento del bicameralismo.

Invece, il legislatore ha prima realizzato la legge elettorale in quanto più semplice da far approvare da una maggioranza parlamentare. Quindi si è dedicato alla riforma costituzionale. Si ha perciò un rovesciamento del processo di produzione normativa. L'entrata in vigore della legge elettorale è stata fissata ad oltre un anno dalla sua approvazione, in modo da permettere l'approvazione della riforma costituzionale, che il legislatore ordinario presumeva potersi compiere entro un breve termine. Sarebbe invece stato più opportuno vincolare l'operatività della nuova legge elettorale al buon esito della riforma costituzionale, piuttosto che ad un termine apparentemente lontano nel tempo come quello del 01.07.2016.

24 Ibidem. 
Infatti la legge di revisione costituzionale è soggetta ad una consultazione referendaria nel mese di ottobre.

In questo modo, dal $1^{\circ}$ luglio 2016 fino al giorno del referendum, la nuova legge per l'elezione della Camera sarà sicuramente in vigore insieme alla vecchia legge per l'elezione del Senato. Inoltre, nel caso in cui l'esito del referendum costituzionale fosse negativo, la coesistenza di queste due leggi sarà definitiva.

Ne deriverebbe quindi la previsione di un premio di maggioranza per la Camera dei deputati, mentre l'elezione del Senato avverrebbe con un sistema proporzionale puro: la disciplina sul premio di maggioranza non è infatti estendibile al Senato, eletto su base regionale secondo l'art. 57 comma 1 Cost.

Questa disciplina elettorale determinerebbe un'irragionevole sacrificio del principio di rappresentatività. La sua contrazione non permetterebbe di conseguire un congruo risultato in termini di governabilità. Alla luce della sentenza della Corte Costituzionale infatti, il premio di maggioranza risulta legittimo e giustificabile solo se riesca ad assicurare la stabilità di governo e una maggioranza ampia. In questo caso tuttavia il risultato non verrebbe conseguito in quanto si formerebbero due differenti maggioranze tra i due rami del Parlamento.

Un secondo profilo di illegittimità costituzionale è rappresentato dalla previsione sui capilista bloccati. Poiché il territorio nazionale è suddiviso in 100 collegi plurinominali, la lista che ottiene il premio di maggioranza può conseguire al massimo cento deputati eletti come capilista e quantomeno 240 eletti con voto di preferenza.

Invece, le liste che dovessero ottenere meno di cento seggi parlamentari sarebbero per lo più rappresentate da deputati eletti come capilista e quindi senza voto di preferenza.

Vista la bassa soglia di sbarramento, molte liste potrebbero superarla ottenendo meno di cento deputati. Pertanto la norma sui capilista bloccati potrebbe contrastare con il principio della libertà di voto (art. 48 Cost.), almeno in relazione agli elettori delle liste di minoranza. Essa impedisce che il rapporto di rappresentanza tra elettori ed eletti si costituisca direttamente e correttamente, limitando la libertà di scelta degli elettori di minoranza nell'elezione dei propri rappresentanti in Parlamento. Inoltre viene indebolito il rapporto 
di responsabilità politica tra elettori ed eletti, sussistendo unicamente un rapporto tra gli eletti e i presentatori delle liste elettorali.

Un ulteriore profilo di illegittimità costituzionale potrebbe essere rappresentato dalla disciplina relativa al ballottaggio: manca infatti la previsione, in caso di secondo turno elettorale, di una soglia minima di voti da raggiungere per ottenere il premio di maggioranza. Si aggiunga che il premio di maggioranza viene attribuito a una lista e non ad una coalizione di liste.

Alla luce di queste considerazioni, è quindi possibile che il premio di maggioranza venga conseguito da una lista che abbia ottenuto il consenso di una percentuale molto ridotta degli aventi diritto al voto. Infatti, essendo il sistema politico italiano pluralistico e fortemente ideologizzato, molti elettori potrebbero non votare nella seconda tornata elettorale, a causa della mancanza di un competitore che corrisponda anche solo lontanamente al proprio orientamento politico.

Senza la previsione di una soglia minima di voti da raggiungere per ottenere il premio di maggioranza, il meccanismo del ballottaggio rischia di determinare un'eccessiva sovra-rappresentanza della lista che avesse conseguito la maggioranza dei suffragi al secondo turno. Inoltre il ballottaggio non garantisce una scelta reale, ma una mera opzione, non risolvendo pertanto i problemi relativi alla carenza di rappresentanza democratica della lista vincitrice.

Si può concludere che le suddette norme perseguono i principi costituzionali della stabilità di governo e dell'efficienza dei processi decisionali nell'ambito parlamentare, senza tuttavia rispettare il vincolo del minor sacrificio possibile degli altri interessi costituzionali protetti: si ha infatti un eccessivo sacrificio del principio di rappresentatività del Parlamento, nonché di quello di libertà di voto.

Per questi motivi, un gruppo di giuristi appartenenti allo stesso comitato che aveva intrapreso la battaglia contro la precedente legge elettorale, presentava dinnanzi a diciotto Tribunali italiani dei ricorsi che esponevano diversi possibili motivi di illegittimità costituzionale della legge 52/2015. Nel mese di febbraio 2016, il Tribunale di Messina rinviava alla Corte costituzionale la questione di illegittimità costituzionale della nuova legge elettorale ${ }^{25}$.

25 Ibidem. 


\section{A legge di revisione costituzionale del 2016}

Come evidenziato nel paragrafo precedente, il processo di riforma dell'assetto istituzionale della Repubblica italiana passa innanzitutto attraverso la legge di revisione costituzionale. La nuova legge elettorale è infatti una semplice norma attuativa della riforma. Ai sensi dell'art. 138 Cost. $^{26}$, il disegno di legge di revisione costituzionale del 2016 veniva approvato la prima volte da entrambe le Camere a maggioranza semplice, dal Senato il 13 ottobre 2015 e dalla Camera l' 11 gennaio 2016. In data 20 gennaio 2016 il Senato approvava per la seconda volta la riforma a maggioranza assoluta, e il giorno 12 aprile lo stesso avveniva alla Camera. Non essendo stata approvata con la maggioranza di due terzi dei componenti di ciascuna Camera, la legge di revisione costituzionale verrà sottoposta a referendum popolare, come richiesto da un congruo numero di parlamentari ai sensi dell'art. 138 Cost. La legge di revisione costituzionale prevede innanzitutto il superamento del bicameralismo perfetto attraverso una riforma strutturale e funzionale del Senato. Modifica inoltre il procedimento di formazione legislativa e la disciplina del referendum. Abolisce le province e modifica il titolo V relativo agli enti locali e ai loro rapporti con lo Stato centrale. Infine modifica il sistema di elezione del Presidente della Repubblica e la nomina dei giudici della Corte Costituzionale da

${ }^{26}$ L'art. 138 Cost. stabilisce l'iter per l'adozione delle leggi di revisione della Costituzione e delle altre leggi costituzionali. Il procedimento per la loro approvazione prevede due deliberazioni successive da parte di ciascuna Camera. Nella prima deliberazione è sufficiente la maggioranza relativa. Decorso un intervallo non minore di tre mesi, può avvenire la seconda votazione, nella quale è richiesta l'approvazione della maggioranza assoluta dei componenti di ciascuna Camera. Tuttavia in questo caso non si tratta di un'approvazione definitiva. Infatti, le leggi così approvate sono sottoposte a referendum popolare quando, entro tre mesi dalla loro pubblicazione, ne facciano domanda un quinto dei membri di una Camera o cinquecentomila elettori o cinque Consigli regionali. La legge sottoposta a referendum non è promulgata, se non è approvata dalla maggioranza dei voti validi. Non si fa luogo a referendum se la legge è stata approvata nella seconda votazione da ciascuna delle Camere a maggioranza di due terzi dei suoi componenti. 
parte del Parlamento. Tale riforma pertanto ha un impatto sulla forma di Stato regionale, ma soprattutto sulla forma di Governo. In particolare, ai fini della presente trattazione rileva la riforma del Senato e il superamento del bicameralismo perfetto. Innanzitutto al Senato è attribuita la funzione di rappresentanza degli enti territoriali, nonché di raccordo tra lo Stato e gli altri enti costitutivi della Repubblica. Il numero di senatori viene ridotto da trecentoquindici a cento: novantacinque sono rappresentativi degli enti territoriali e cinque di nomina presidenziale.

Mutano inoltre le modalità di elezione del Senato, che non si svolge più a suffragio universale e diretto, ma con un'elezione di secondo grado da parte delle assemblee degli enti territoriali. In particolare, i novantacinque senatori sono eletti con metodo proporzionale dai consigli regionali e dalle province autonome di Trento e Bolzano tra i propri membri e uno tra i sindaci dei comuni del proprio territorio.

I restanti cinque senatori sono nominati dal Presidente della Repubblica tra coloro che si sono distinti per meriti nel campo scientifico, artistico, letterario e sociale. Essi durano in carica sette anni e non possono essere nuovamente nominati. Rimane invece immutata la carica a senatore a vita per gli ex Presidenti della Repubblica.

Il Senato diviene un organo a rinnovo parziale, non sottoposto a scioglimento, poiché la durata dei senatori eletti coincide con quella degli organi delle istituzioni territoriali dai quali sono stati eletti. Ogni regione deve avere almeno due senatori, le province autonome ne hanno due a testa. La ripartizione dei seggi viene fatta in base alla popolazione risultante dall'ultimo censimento. E' comunque delegata alla legge tutta la normativa di attuazione del sistema come ad esempio la normativa in materia di sostituzione dei senatori in caso di cessazione della carica elettiva regionale o locale. In definitiva la riforma realizza un bicameralismo differenziato. La Camera dei deputati rappresenta la nazione ed è esclusiva titolare del rapporto fiduciario con il Governo, nonché effettiva titolare del potere legislativo.

Il Senato invece rappresenta gli enti territoriali e svolge la funzione di raccordo tra lo Stato, gli altri enti costitutivi della Re- 
pubblica e l'Unione europea. Inoltre concorre con l'altra Camera nell'esercizio del potere legislativo esclusivamente nei casi e secondo le modalità stabilite dal nuovo art. 70 , comma 1 della Costituzione ${ }^{27}$.

Nei restanti casi le leggi vengono approvate soltanto dalla Camera dei deputati con un procedimento legislativo monocamerale (art. 70 comma 2).

Il Senato effettua poi la valutazione delle politiche pubbliche e dell'attività delle pubbliche amministrazioni.

Si evidenzia quindi che il bicameralismo perfetto viene superato attraverso la fine della parità legislativa tra le due Camere e il mantenimento della funzione legislativa piena soltanto in capo alla Camera dei deputati ${ }^{28}$.

\section{Criticita' legge di revisione costituzionale del 2016}

L'obiettivo della riforma istituzionale è quello di superare un sistema di bicameralismo perfetto, retaggio del passato, non più attuale nei sistemi statali odierni. A tal proposito si è optato per un mutamento strutturale e funzionale della Camera Alta, piuttosto che per la sua eliminazione dall'ordinamento. Tuttavia la riforma non tiene conto dell'importante ruolo che, al momento della redazione

${ }^{27}$ In particolare il Senato esercita la funzione legislativa con la Camera dei deputati nei seguenti casi: per le leggi di revisione della Costituzione e le altre leggi costituzionali, per le leggi di attuazione delle disposizioni costituzionali concernenti la tutela delle minoranze linguistiche, i referendum popolari, le altre forme di consultazione di cui all'articolo 71, per le leggi che determinano l'ordinamento, la legislazione elettorale, gli organi di governo, le funzioni fondamentali dei Comuni e delle Città metropolitane e le disposizioni di principio sulle forme associative dei Comuni, per la legge che stabilisce le norme generali, le forme e i termini della partecipazione dell'Italia alla formazione e all'attuazione della normativa e delle politiche dell'Unione europea, per quella che determina i casi di ineleggibilità e di incompatibilità con l'ufficio di senatore e altre tipologie di leggi previste sempre dalla Costituzione.

28 G. Azzariti, Le modifiche costituzionali in sintesi. Scheda/commento del Prof. Azzariti, www.coordinamentodemocraziacostituzionale.net (access: 15.12.2015). 
della Costituzione, l'Assemblea Costituente attribuiva anche al Senato al fine di garantire l'equilibrio costituzionale tra i poteri ${ }^{29}$. I Costituenti infatti garantivano la centralità al Parlamento al fine di evitare derive autoritarie dell'esecutivo e un procedimento di formazione legislativa più ponderato, dovendo le leggi essere approvate nello stesso testo da entrambe le Camere. Perciò, facendo venire meno questo contrappeso, la riforma indebolisce il potere parlamentare a favore dell'esecutivo: da una parte con la riduzione del numero dei senatori e la diminuzione delle funzioni del Senato, dall'altra con l'ampio premio di maggioranza previsto alla Camera dei deputati dalla legge elettorale. Queste riforme istituzionali pertanto, in nome della governabilità e della rapidità dei meccanismi legislativi, spostano di fatto il baricentro verso il premier nonché segretario nazionale del partito di maggioranza: egli si trova in una posizione di controllo del partito e attraverso di esso può operare nelle istituzioni, sia a livello nazionale sia a livello locale. Infatti alla Camera il suo partito ottiene il 55\% dei seggi e, se coeso, può autonomamente svolgere la funzione legislativa ordinaria, senza altri contrappesi.

Si evidenzia inoltre che nelle riunioni del Parlamento in seduta comune la maggioranza presente alla Camera ha un'importanza fondamentale, mentre il Senato ha un potere decisionale marginale: infatti, l'eccessiva differenza numerica dei seggi che compongono la Camera e il Senato è tale da rendere irrilevante la presenza dei senatori nelle elezioni del Parlamento in seduta comune. Come affermato, al Senato si è attribuita una connotazione diversa al fine di giustificare l'assetto bicamerale: questo organo rappresenta quindi gli interessi degli enti territoriali. Tuttavia per i senatori viene mantenuto il divieto di mandato imperativo, che vanifica questa finalità rappresentativa e fa rivivere la logica dell'appartenenza politica.

Appare infatti più coerente con la predetta finalità rappresentativa vincolare il voto dei senatori alle indicazioni dell'ente territoriale che li ha eletti, come avviene, ad esempio, nel Bundesrat tedesco,

29 Il bilanciamento tra i poteri, principio fondamentale negli ordinamenti democratici, consiste in una serie di meccanismi politico-istituzionali finalizzati a mantenere l'equilibrio tra i vari poteri all'interno di uno Stato. 
dove per l'appunto i membri di un medesimo Länder sono tenuti a votare unitariamente, sulla base di una predeterminata scelta dell'ente rappresentato. In questo modo si evita che i rappresentanti della seconda Camera si aggreghino secondo logiche partitiche, tipiche delle Camere politiche, e si agevola invece l'espressione dei variabili interessi territoriali, secondo la funzione delle Camere federali. Al contrario la presente riforma costituzionale, con il divieto del mandato imperativo, agevola l'influenza del partito e del suo segretario nazionale sulle decisioni dei consigli regionali e del Senato.

In definitiva, la legge di revisione costituzionale elimina i contropoteri esterni alla Camera senza compensarli con altri interni, e sposta quindi l'equilibrio dei poteri costituzionali a favore dell'esecutivo: il Parlamento risulterà pertanto sottomesso alle iniziative del governo, per l'effetto congiunto della riforma costituzionale e della legge elettorale ${ }^{30}$.

\section{Conclusioni}

Si può notare come le riforme elettorali degli ultimi venticinque anni abbiano principalmente tentato di rafforzare la stabilità dell'esecutivo e di semplificare il pluralismo politico, contraendo inevitabilmente l'indice di rappresentatività, ossia la corrispondenza tra i voti ottenuti da ogni lista e i seggi effettivamente conseguiti. Il processo di rafforzamento del potere esecutivo ha raggiunto il punto più alto con la recentissima legge di revisione costituzionale del 2016, la quale ha tuttavia ridotto la centralità del Parlamento e ha spostato l'equilibrio tra i poteri costituzionali a favore del governo. Come affermato dalla Corte Costituzionale, l'esigenza della governabilità richiede inevitabilmente una riduzione del grado di rappresentatività, ma non può tuttavia giustificare uno sbilanciamento istituzionale a favore dell'esecutivo: in un ordinamento democratico, il Parlamento deve essere il luogo del compromesso

30 A. Pace, Le insuperabili criticità della riforma costituzionale Renzi, www. coordinamentodemocraziacostituzionale.net (access: 21.02.016). 
politico, dell'incontro tra le istanze e gli interessi delle numerose forze politiche che rappresentano il pluralismo sociale.

Sarebbe infatti assolutamente inopportuno garantire un esecutivo stabile per tutta la durata della legislatura, in virtù di uno sbilanciamento di poteri in suo favore. Risulta pertanto necessario che il principio di governabilità venga perseguito nel rispetto dei principi fondamentali degli ordinamenti democratici: in primo luogo il check and balance, ossia il bilanciamento e l'equilibrio tra i poteri, in secondo luogo la valorizzazione del pluralismo politico all'interno dell'organo assembleare, che deve essere punto di incontro e di sintesi dei numerosi interessi sociali.

\section{STRESZCZENIE}

Ewolucja włoskiego systemu wyborczego od narodzin republiki do reformy z 2015 r.

Niniejszy artykuł ma na celu ukazanie procesu przekształcania się włoskiego ustawodastwa wyborczego, od okresu tuż po II wojnie światowej aż po czasy współczesne. Punktem wyjścia szczegółowej analizy tytułowego zagadnienia jest omówienie przepisów Konstytucji Republiki Włoskiej z 1947 r. odnoszących się do problematyki prawa wyborczego. Autor ukazuje także specyfikę ewolucji włoskiej ordynacji wyborczej, począwszy od systemu proporcjonalnego, kończąc na współczesnych systemach większościowych. Autor wiele miejsca poświęca także analizie ostatnich propozycji legislacyjnych dotyczących włoskiego sysmetu wyborczego.

Słowa kluczowe: Republika Włoska; system wyborczy; Izba Deputowanych; senat; wybory

\section{SUMMARY}

The evolution of the Italian electoral system from the rise of the republic to the reform in 2015

The present article is aimed at show the transformation of the Italian electoral legislation, starting from the period just after the Second World War until contemporary times. The point of departure for the present 
analyses is the discussion around the provisions of the Italian Republic's Constitution of 1947, related to the issues of electoral right. The author also shows the specific evolution of italian electoral law, starting from a proportional system and up to the contemporary majority system. The author also dedicates much space to an analysis of the recent legislative proposals about the Italian electoral system.

Keywords: Italian Republic; electoral system; Chamber of Deputies; Senate; elections

\section{BIBLIOGRAFIA}

Azzariti G., Le modifiche costituzionali in sintesi. Scheda/commento del Prof. Azzariti, www.coordinamentodemocraziacostituzionale.net (access: 15.12.2015).

Bin R., Pitruzzella G., Diritto costituzionale, Giappichelli, Torino 2013.

Dickmann R., A proposito dell'Italicum. Prime osservazioni sul nuovo sistema elettorale di cui alla legge n. 52 del 6 maggio 2015, www.forumcostituzionale.it (access: 03.06.2015).

Fusaro C., Le regole della transizione - La nuova legislazione elettorale italiana, Il Mulino 1995.

Pace A., Le insuperabili criticità della riforma costituzionale Renzi, www. coordinamentodemocraziacostituzionale.net (access: 21.02.2016).

Pastore F., Partiti e movimenti politici nel momento elettorale sotto la vigenza dell'Italicum, www.dirittifondamentali.it (access: 11.05.2015).

Tosi D. E., “Forme di governo", in: Diritto costituzionale dei paesi dell'Unione Europea, a cura di E. Palici Di Suni, Cedam, Padova 2007. 
